\title{
Von der Ethik zum Recht ... und wieder zurück?
}

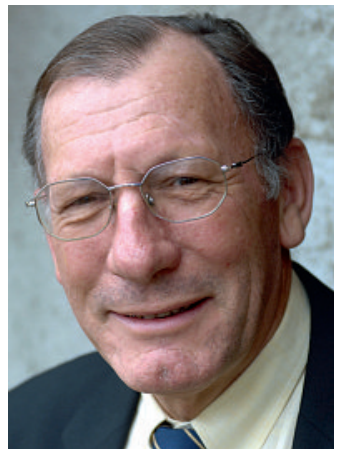

Jean Martin
«Der geistige Wandel, die gewachsene Bedeutung von Gesundheit und Medizin sowie die Entwicklung neuer Technologien, die die Integrität des Körpers bedrohen und die Zusammenhänge der Abstammung unscharf werden lassen, geben Anlass zu ethischen Überlegungen, die vom Gesetzgeber aufgegriffen und im Recht verankert werden», schreibt der bekannte Bordelaiser Professor Bernard Hoerni [1]. Sein Buch ist sehr empfehlenswert.

In jüngerer Vergangenheit wurden in verschiedensten Bereichen gesetzliche Regelungen geschaffen, wo es zuvor nur berufsständische Empfehlungen gab - oder auch gar keine Vorgaben: künstliche Fortpflanzung, Transplantionsmedizin, Stammzellenforschung ... Wir haben am 7. März über einen Verfassungsartikel zur Forschung abgestimmt. Die Festlegung derartiger Regeln ist notwendig, doch auch die Berufsethik muss weiterhin Raum und Gewicht haben, denn ein Gesetz ist von seinem Wesen her starr und dadurch nicht immer das richtige Instrument.

Es kann auch vorkommen, dass eine gesetzliche Regelung erst Unsicherheit stiftet. Ein Beispiel ist das neue Erwachsenenschutzrecht, das Ende 2008 verabschiedet wurde und mit dem die Patientenverfügung (PV) Einzug in das Zivilgesetzbuch (ZGB) gehalten hat. Die PV ist ein sinnvolles Instrument, das unseren Mitbürgern noch nähergebracht werden muss [2,3]. Das Gesetz schreibt vor, dass eine PV in Schriftform mit Datum und Unterschrift vorliegen muss (Artikel 371). Das Streben nach Präzision ist verständlich, aber nicht unproblematisch: Es könnte den absolut nicht wünschenswerten Effekt nach sich ziehen, dass einige Ärzte - oder andere Betreuungspersonen - daraus folgern, dass eine mündlich geäusserte Willensbekundung eines urteilsfähigen Patienten keinen Wert mehr hat oder nicht mehr bindend ist. Aus ethischer Sicht jedoch kann kein Arzt sich ohne schwerwiegenden Grund über klare Anweisungen des Patienten hinwegsetzen, auch wenn es keine schriftlichen Verfügungen sind. Wenn dieser Effekt einträte, würde ein Fortschritt in der Gesetzgebung erkauft mit einer qualitativen Herabsetzung des Arzt-Patienten-Verhältnisses. Allerdings muss man gerechterweise erwähnen, dass der Bundesrat in seiner Botschaft schreibt: «Wird die Form nicht gewahrt, ist die Patientenverfügung rechtlich nicht einfach unbeachtlich.»

Ein weiterer wichtiger Punkt: Bisher entschied, wenn der Wille des Patienten nicht bekannt war, in letzter Instanz der Arzt gemeinsam mit dem Behandlungsteam über das weitere Vorgehen. Jedoch betont der Bundesrat: «Nach dem Europäischen Übereinkommen über Biomedizin ist es nicht zulässig, den Angehörigen lediglich ein Anhörungsrecht zu geben und die Ärzteschaft oder deren Hilfspersonen für ihre Patientin oder ihren Patienten selber entscheiden zu lassen, wie gewisse kantonale Gesetze dies heute vorsehen.» Im ZGB werden die Personen genau aufgezählt, die berechtigt sind, einen Patienten zu vertreten, wenn dieser keine Patientenverfügung verfasst hat, und es wird eine kaskadenartige Ordnung dieser Vertreter festgelegt (Vertretungsperson, Beistand, Partner usw.). Auch diese Änderung könnte zu Schwierigkeiten und Schuldzuweisungen führen: Es ist nicht leicht, im Namen einer nahestehenden schwerkranken Person eine Entscheidung von grosser Tragweite zu fällen, zum Beispiel über den Verzicht auf eine Behandlungsmassnahme. Wobei hiermit in keiner Weise der ärztliche «Machtverlust» beklagt, sondern lediglich die Frage aufgeworfen werden kann, ob die Änderung optimal ist.

Eine weitere Entwicklung, bei der man sich fragt, ob sich nicht ein gewisser «Wildwuchs» an Kontrolle und Überwachung ausbreitet, betrifft das Bundesgesetz über die Transplantation und dessen Anwendungsbestimmungen, die dem medizinischen Personal Teile seines Entscheidungsspektrums entziehen, die es sich gern bewahrt hätte.

Während meiner Tätigkeit als Kantonsarzt habe ich mich für die Stärkung der Patientenrechte eingesetzt und auch dafür, dass sie im Gesetz verankert werden - das ist sinnvoll, weil das von den Parlamenten verabschiedete öffentliche Recht für jedermann bindend ist. Wenn ich jedoch einige aktuelle Fragen betrachte, erscheint es wünschenswert, dass auch der medizinischen Ethik weiterhin eine hinreichende Bedeutung beigemessen wird. Ich höre schon den Einwand laut werden, dass Ärzte nicht unfehlbar sind, was natürlich stimmt, und dass ihre Berufsethik nicht demokratisch legitimiert ist. Doch an den Stellen, an denen diese genausogut wie oder besser als das Gesetz ist, flexibler, verdient sie aufgewertet $\mathrm{zu}$ werden. Gerade wenn es um Privates geht, ist die legitime Rolle des Gesetzes begrenzt; ein Übermass an Gesetzen erzeugt den falschen Eindruck, dass der Staat besser wisse als der Einzelne, was gut für diesen ist.

Eine Gefahr liegt darin, dass detaillierte Vorschriften und externe Kontrollen diejenigen aus der Verantwortung entlassen, die nach ihrem besten Wissen den individuellen Einzelfall des Patienten behandeln.

Kurz gesagt: Es gilt, die gesetzlich festgeschriebenen Rechte und Pflichten in Einklang zu bringen mit dem notwendigen gegenseitigen Vertrauen innerhalb der Arzt-Patienten-Beziehung, das kein paternalistisches, sondern ein gleichberechtigtes Verhältnis ist, mit modernen Facetten von Transparenz und Wahrhaftigkeit.

Jean Martin* 